\title{
Unitarily invariant norm inequalities involving Heron and Heinz means
}

Haisong Cao* and Junliang Wu

${ }^{*}$ Correspondence: hscao123@163.com

College of Mathematics and Statistics, Chongqing University,

Chongqing, 401331, P.R. China

\begin{abstract}
In this paper, we present some new inequalities for unitarily invariant norms involving Heron and Heinz means for matrices, which generalize the result of Theorem 2.1 (Fu and $\mathrm{He}$ in J. Math. Inequal. 7(4):727-737, 2013) and refine the inequality of Theorem 6 (Zhan in SIAM J. Matrix Anal. Appl. 20: 466-470, 1998). Our results are a refinement and a generalization of some existing inequalities.
\end{abstract}

MSC: 47A30; $15 \mathrm{~A} 60$

Keywords: unitarily invariant norm; positive definite matrices; convex function; Heron means; Heinz means

\section{Introduction}

Throughout, let $M_{m, n}$ be the space of $m \times n$ complex matrices and $M_{n}=M_{n, n}$.

A norm $\|\cdot\|$ is called unitarily invariant norm if $\|U A V\|=\|A\|$ for all $A \in M_{n}$ and for all unitary matrices $U, V \in M_{n}$. Two classes of unitarily invariant norms are especially important. The first is the class of the Ky Fan $k$-norm $\|\cdot\|_{(k)}$, defined as

$$
\|A\|_{(k)}=\sum_{j=1}^{k} s_{j}(A), \quad k=1, \ldots, n,
$$

where $s_{i}(A)(i=1, \ldots, n)$ are the singular values of $A$ with $s_{1}(A) \geq \cdots \geq s_{n}(A)$, which are the eigenvalues of the positive semidefinite matrix $|A|=\left(A^{*} A\right)^{\frac{1}{2}}$, arranged in decreasing order and repeated according to multiplicity. The second is the class of the Schatten $p$ norm $\|\cdot\|_{(p)}$, defined as

$$
\|A\|_{p}=\left(\sum_{j=1}^{n} s_{j}^{p}(A)\right)^{\frac{1}{p}}=\left(\operatorname{tr}|A|^{p}\right)^{\frac{1}{p}}, \quad 1 \leq p<\infty .
$$

For two nonnegative real numbers $a$ and $b$, the Heinz mean and Heron mean in the parameter $v, 0 \leq v \leq 1$, are defined, respectively, as

$$
\begin{aligned}
& H_{v}(a, b)=\frac{a^{v} b^{1-v}+a^{1-v} b^{v}}{2}, \\
& F_{\alpha}(a, b)=(1-\alpha) \sqrt{a b}+\alpha \frac{a+b}{2} .
\end{aligned}
$$

C) 2014 Cao and Wu; licensee Springer. This is an Open Access article distributed under the terms of the Creative Commons Attribution License (http://creativecommons.org/licenses/by/2.0), which permits unrestricted use, distribution, and reproduction in any medium, provided the original work is properly cited. 
Note that $H_{0}(a, b)=H_{1}(a, b)=\frac{a+b}{2}$ (the arithmetic mean of $a$ and $b$ ) and $H_{\frac{1}{2}}(a, b)=\sqrt{a b}$ (the geometric mean of $a$ and $b$ ). It is easy to see that as a function of $v, H_{v}(a, b)$ is convex, attains its minimum at $v=\frac{1}{2}$, and attains its maximum at $v=0$ and $v=1$.

The operator version of the Heinz mean [1] asserts that if $A, B$ and $X$ are operators on a complex separable Hilbert space such that $A$ and $B$ are positive, then for every unitarily invariant norm $\|\cdot\|$, the function $g(v)=\left\|A^{v} X B^{1-v}+A^{1-v} X B^{v}\right\|$ is convex on $[0,1]$, attains its minimum at $v=\frac{1}{2}$, and attains its maximum at $v=0$ and $v=1$. Moreover, the operator version of the Heron mean [2] asserts that $f(\alpha)=\left\|(1-\alpha) A^{\frac{1}{2}} X B^{\frac{1}{2}}+\alpha\left(\frac{A X+X B}{2}\right)\right\|$.

Let $A, B, X \in M_{n}, A, B$ are positive definite, Kaur and Singh [2] have proved the following inequalities for any unitarily invariant norm $\|\cdot\|$ :

$$
\frac{1}{2}\left\|A^{v} X B^{1-v}+A^{1-v} X B^{v}\right\| \leq\left\|(1-\alpha) A^{\frac{1}{2}} X B^{\frac{1}{2}}+\alpha\left(\frac{A X+X B}{2}\right)\right\|
$$

and

$$
\left\|A^{\frac{1}{2}} X B^{\frac{1}{2}}\right\| \leq \frac{1}{2}\left\|A^{\frac{2}{3}} X B^{\frac{1}{3}}+A^{\frac{1}{3}} X B^{\frac{2}{3}}\right\| \leq \frac{1}{2+t}\left\|A X+t A^{\frac{1}{2}} X B^{\frac{1}{2}}+X B\right\|,
$$

where $\frac{1}{4} \leq v \leq \frac{3}{4}, \alpha \in\left[\frac{1}{2}, \infty\right)$ and $t \in(-2,2]$.

Replacing $A, B$ by $A^{2}, B^{2}$ in (1.1) and (1.2), then putting $u=2 v$, the following inequalities hold:

$$
\frac{1}{2}\left\|A^{u} X B^{2-u}+A^{2-u} X B^{u}\right\| \leq\left\|(1-\alpha) A X B+\alpha\left(\frac{A^{2} X+X B^{2}}{2}\right)\right\|
$$

and

$$
\|A X B\| \leq \frac{1}{2}\left\|A^{\frac{4}{3}} X B^{\frac{2}{3}}+A^{\frac{2}{3}} X B^{\frac{4}{3}}\right\| \leq \frac{1}{t+2}\left\|A^{2} X+t A X B+X B^{2}\right\|,
$$

where $\frac{1}{2} \leq u \leq \frac{3}{2}, \alpha \in\left[\frac{1}{2}, \infty\right)$ and $t \in(-2,2]$.

Zhan proved in [3] that if $A, B, X \in M_{n}$, such that $A, B$ are positive semidefinite, then

$$
\left\|A^{u} X B^{2-u}+A^{2-u} X B^{u}\right\| \leq \frac{2}{t+2}\left\|A^{2} X+t A X B+X B^{2}\right\|
$$

for $\frac{1}{2} \leq u \leq \frac{3}{2}$ and $t \in(-2,2]$.

Let $A, B, X \in M_{n}$, such that $A, B$ are positive semidefinite, for $\frac{1}{2} \leq u \leq \frac{3}{2}$ and $t \in(-2,2]$;

Fu et al. in [4] proved that

$$
\begin{aligned}
& 2\|A X B\|+2\left(\int_{\frac{1}{2}}^{\frac{3}{2}}\left\|A^{r} X B^{2-r}+A^{2-r} X B^{r}\right\| d r-2\|A X B\|\right) \\
& \leq \frac{2}{t+2}\left\|A^{2} X+t A X B+X B^{2}\right\| .
\end{aligned}
$$

Recently, Kaur et al. [5], He et al. [6] and Bakherad et al. [7] have studied similar topics. For the sake of convenience, we set

$$
g(u)=\left\|\frac{A^{u} X B^{2-u}+A^{2-u} X B^{u}}{2}\right\| .
$$


In Section 2, we will generalize and refine some existing inequalities for unitarily invariant norms involving Heron and Heinz means for matrices and present some new refinements of the inequalities above.

\section{Main results}

In this section, we firstly utilize the convexity of the function $g(u)$ to obtain a unitarily invariant norms inequality that leads to another version of the inequality (1.6), which is also the refinement of the inequality (1.5).

To obtain the results, we need the following lemma on convex functions $[8,9]$.

Lemma 2.1 Let $f$ be a real valued continuous convex function on an interval $[a, b]$ which contains $\left(x_{1}, x_{2}\right)$. Then for $x_{1} \leq x \leq x_{2}$, we have

$$
f(x) \leq \frac{f\left(x_{2}\right)-f\left(x_{1}\right)}{x_{2}-x_{1}} x-\frac{x_{1} f\left(x_{2}\right)-x_{2} f\left(x_{1}\right)}{x_{2}-x_{1}} .
$$

Theorem 2.2 Let $A, B, X \in M_{n}$, such that $A, B$ are positive semidefinite. Then for any unitarily invariant norm $\|\cdot\|, \frac{1}{2} \leq u \leq \frac{3}{2}$ and $\alpha \in\left[\frac{1}{2}, \infty\right)$,

$$
\begin{aligned}
\left\|A^{u} X B^{2-u}+A^{2-u} X B^{u}\right\| \leq & 2\left(4 r_{0}-1\right)\|A X B\| \\
& +4\left(1-2 r_{0}\right)\left\|(1-\alpha) A X B+\alpha\left(\frac{A^{2} X+X B^{2}}{2}\right)\right\|,
\end{aligned}
$$

where $r_{0}=\min \left[\frac{u}{2}, 1-\frac{u}{2}\right]$.

Proof For $\frac{1}{2} \leq u \leq 1$, by the convexity of the function $g(u)$ and Lemma 2.1, presented above, we have

$$
g(u) \leq \frac{g(1)-g\left(\frac{1}{2}\right)}{\frac{1}{2}} u-\frac{\left.\frac{1}{2} g(1)-g\left(\frac{1}{2}\right)\right)}{\frac{1}{2}},
$$

which implies

$$
g(u) \leq 2(1-u) g\left(\frac{1}{2}\right)+(2 u-1) g(1) .
$$

By (1.3) and (2.2), we have

$$
\left\|A^{u} X B^{2-u}+A^{2-u} X B^{u}\right\| \leq 4(1-u)\left\|(1-\alpha) A X B+\alpha\left(\frac{A^{2} X+X B^{2}}{2}\right)\right\|+2(2 u-1)\|A X B\| .
$$

So,

$$
\begin{aligned}
\left\|A^{u} X B^{2-u}+A^{2-u} X B^{u}\right\| \leq & 2\left(4 r_{0}-1\right)\|A X B\| \\
& +4\left(1-2 r_{0}\right)\left\|(1-\alpha) A X B+\alpha\left(\frac{A^{2} X+X B^{2}}{2}\right)\right\| .
\end{aligned}
$$


For $1 \leq u \leq \frac{3}{2}$, by the convexity of the function $g(u)$ and Lemma 2.1, presented above, we have

$$
g(u) \leq \frac{g\left(\frac{3}{2}\right)-g(1)}{\frac{1}{2}} u-\frac{g\left(\frac{3}{2}\right)-\frac{3}{2} g(1)}{\frac{1}{2}},
$$

which implies

$$
g(u) \leq(3-2 u) g(1)+2(u-1) g\left(\frac{3}{2}\right) .
$$

By (1.3) and (2.4), we have

$$
\begin{aligned}
\left\|A^{u} X B^{2-u}+A^{2-u} X B^{u}\right\| \leq & 2(3-2 u)\|A X B\| \\
& +4(u-1)\left\|(1-\alpha) A X B+\alpha\left(\frac{A^{2} X+X B^{2}}{2}\right)\right\| .
\end{aligned}
$$

So,

$$
\begin{aligned}
\left\|A^{u} X B^{2-u}+A^{2-u} X B^{u}\right\| \leq & 2\left(4 r_{0}-1\right)\|A X B\| \\
& +4\left(1-2 r_{0}\right)\left\|(1-\alpha) A X B+\alpha\left(\frac{A^{2} X+X B^{2}}{2}\right)\right\| .
\end{aligned}
$$

By (2.3) and (2.5), for $\frac{1}{2} \leq u \leq \frac{3}{2}, \alpha \in\left[\frac{1}{2}, \infty\right)$ and $r_{0}=\min \left[\frac{u}{2}, 1-\frac{u}{2}\right]$, we have the following equivalent inequality:

$$
\begin{aligned}
\left\|A^{u} X B^{2-u}+A^{2-u} X B^{u}\right\| \leq & 2\left(4 r_{0}-1\right)\|A X B\| \\
& +4\left(1-2 r_{0}\right)\left\|(1-\alpha) A X B+\alpha\left(\frac{A^{2} X+X B^{2}}{2}\right)\right\| .
\end{aligned}
$$

The proof is completed.

Remark 2.3 With a simple computation between the upper bounds in (1.3) and (2.1), obviously we have

$$
\begin{aligned}
\| & (1-\alpha) A X B+\alpha\left(\frac{A^{2} X+X B^{2}}{2}\right) \| \\
& \quad-\left(4 r_{0}-1\right)\|A X B\|-2\left(1-2 r_{0}\right)\left\|(1-\alpha) A X B+\alpha\left(\frac{A^{2} X+X B^{2}}{2}\right)\right\| \\
= & \left(4 r_{0}-1\right)\left\|(1-\alpha) A X B+\alpha\left(\frac{A^{2} X+X B^{2}}{2}\right)\right\|-\left(4 r_{0}-1\right)\|A X B\| \\
= & \left(4 r_{0}-1\right)\left(\left\|(1-\alpha) A X B+\alpha\left(\frac{A^{2} X+X B^{2}}{2}\right)\right\|-\|A X B\|\right) \\
> & 0 .
\end{aligned}
$$

Thus the inequality (2.1) is a refinement of the inequality (1.3). 
Now, we present a refinement of the inequality $\|A X B\| \leq\left\|(1-\alpha) A X B+\alpha\left(\frac{A^{2} X+X B^{2}}{2}\right)\right\|$.

Theorem 2.4 Let $A, B, X \in M_{n}$, such that $A, B$ are positive semidefinite. Then for any unitarily invariant norm $\|\cdot\|, \frac{1}{2} \leq u \leq \frac{3}{2}$, and $\alpha \in\left[\frac{1}{2}, \infty\right)$, we have

$$
\begin{aligned}
& 2\|A X B\|+2\left(\int_{\frac{1}{2}}^{\frac{3}{2}}\left\|A^{u} X B^{2-u}+A^{2-u} X B^{u}\right\| d u-2\|A X B\|\right) \\
& \leq\left\|(1-\alpha) A X B+\alpha\left(\frac{A^{2} X+X B^{2}}{2}\right)\right\|,
\end{aligned}
$$

where $r_{0}=\min \left[\frac{u}{2}, 1-\frac{u}{2}\right]$.

Proof For $\frac{1}{2} \leq u \leq 1$, from Theorem 2.2, we have

$$
\begin{aligned}
\left\|A^{u} X B^{2-u}+A^{2-u} X B^{u}\right\| \leq & 4(1-u)\left\|(1-\alpha) A X B+\alpha\left(\frac{A^{2} X+X B^{2}}{2}\right)\right\| \\
& +2(2 u-1)\|A X B\| .
\end{aligned}
$$

By integrating both sides of the inequality above, we have

$$
\begin{aligned}
& \int_{\frac{1}{2}}^{1}\left\|A^{u} X B^{2-u}+A^{2-u} X B^{u}\right\| d u \\
& \leq 4\left\|(1-\alpha) A X B+\alpha\left(\frac{A^{2} X+X B^{2}}{2}\right)\right\| \int_{\frac{1}{2}}^{1}(1-u) d u \\
& \quad+2\|A X B\| \int_{\frac{1}{2}}^{1}(2 u-1) d u \\
& =\frac{1}{2}\left\|(1-\alpha) A X B+\alpha\left(\frac{A^{2} X+X B^{2}}{2}\right)\right\|+\frac{1}{2}\|A X B\| .
\end{aligned}
$$

For $1 \leq u \leq \frac{3}{2}$, from Theorem 2.2, we have

$$
\begin{aligned}
\left\|A^{u} X B^{2-u}+A^{2-u} X B^{u}\right\| \leq & 2(3-2 u)\|A X B\| \\
& +4(u-1)\left\|(1-\alpha) A X B+\alpha\left(\frac{A^{2} X+X B^{2}}{2}\right)\right\| .
\end{aligned}
$$

Similarly, by integrating both sides of the inequality above, we have

$$
\begin{aligned}
& \int_{1}^{\frac{3}{2}}\left\|A^{u} X B^{2-u}+A^{2-u} X B^{u}\right\| d u \\
& \leq 2\|A X B\| \int_{1}^{\frac{3}{2}}(3-2 u) d u \\
& \quad+4\left\|(1-\alpha) A X B+\alpha\left(\frac{A^{2} X+X B^{2}}{2}\right)\right\| \int_{1}^{\frac{3}{2}}(2 u-1) d u \\
& =\frac{1}{2}\left\|(1-\alpha) A X B+\alpha\left(\frac{A^{2} X+X B^{2}}{2}\right)\right\|+\frac{1}{2}\|A X B\| .
\end{aligned}
$$


It follows from (2.7) and (2.8) that

$$
\int_{\frac{1}{2}}^{\frac{3}{2}}\left\|A^{u} X B^{2-u}+A^{2-u} X B^{u}\right\| d u \leq\left\|(1-\alpha) A X B+\alpha\left(\frac{A^{2} X+X B^{2}}{2}\right)\right\|+\|A X B\|
$$

which is equivalent to

$$
\begin{aligned}
& 2\|A X B\|+2\left(\int_{\frac{1}{2}}^{\frac{3}{2}}\left\|A^{u} X B^{2-u}+A^{2-u} X B^{u}\right\| d u-2\|A X B\|\right) \\
& \leq 2\left\|(1-\alpha) A X B+\alpha\left(\frac{A^{2} X+X B^{2}}{2}\right)\right\| .
\end{aligned}
$$

The proof is completed.

Remark 2.5 Obviously,

$$
\int_{\frac{1}{2}}^{\frac{3}{2}}\left\|A^{u} X B^{2-u}+A^{2-u} X B^{u}\right\| d u-2\|A X B\| \geq 0
$$

Thus, the inequality (2.6) is a refinement of the inequality $\|A X B\| \leq \|(1-\alpha) A X B+$ $\alpha\left(\frac{A^{2} X+X B^{2}}{2}\right) \|$.

Taking $\alpha=\frac{2}{t+2}(-2<t \leq 2)$, the following corollaries are obtained.

Corollary 2.6 Let $A, B, X \in M_{n}$, such that $A, B$ are positive semidefinite. Then for any unitarily invariant norm $\|\cdot\|$ and $\frac{1}{2} \leq u \leq \frac{3}{2}$,

$$
\begin{aligned}
\left\|A^{u} X B^{2-u}+A^{2-u} X B^{u}\right\| \leq & 2\left(4 r_{0}-1\right)\|A X B\| \\
& +\frac{4\left(1-2 r_{0}\right)}{t+2}\left\|A^{2} X+t A X B+X B^{2}\right\|,
\end{aligned}
$$

where $r_{0}=\min \left[\frac{u}{2}, 1-\frac{u}{2}\right]$ and $-2<t \leq 2$.

Corollary 2.7 Let $A, B, X \in M_{n}$, such that $A, B$ are positive semidefinite. Then for any unitarily invariant norm $\|\cdot\|, \frac{1}{2} \leq u \leq \frac{3}{2}$,

$$
\begin{aligned}
& 2\|A X B\|+2\left(\int_{\frac{1}{2}}^{\frac{3}{2}}\left\|A^{u} X B^{2-u}+A^{2-u} X B^{u}\right\| d u-2\|A X B\|\right) \\
& \leq \frac{2}{t+2}\left\|A^{2} X+t A X B+X B^{2}\right\|
\end{aligned}
$$

where $r_{0}=\min \left[\frac{u}{2}, 1-\frac{u}{2}\right]$ and $-2<t \leq 2$.

Thus, on the one hand, the inequality (2.9) is a refinement of the inequality $\|A X B\| \leq$ $\frac{1}{t+2}\left\|A^{2} X+t A X B+X B^{2}\right\|$, and also another version of the inequality (1.6); on the other hand, the inequality (2.10) is just the inequality proved in [4], so the inequality (2.6) presented in Theorem 2.4 is also the generalization of the inequality proved in [4]. 
Competing interests

The authors declare that they have no competing interests. $\mathrm{HC}$ is responsible for all the whole of the article appearing.

\section{Authors' contributions}

JW carried out the matrix operator theory studies and participated in the conception and design. HC conceived of the study, participated in its design and drafting the manuscript. All authors read and approved the final manuscript.

\section{Acknowledgements}

The authors wish to express their heartfelt thanks to the referees for their detailed and helpful suggestions for revising the manuscript.

Received: 16 April 2014 Accepted: 4 July 2014 Published: 18 August 2014

\section{References}

1. Kittaneh, F: On the convexity of the Heinz means. Integral Equ. Oper. Theory 68, 519-527 (2010)

2. Kaur, R, Singh, M: Complete interpolation of matrix versions of Heron and Heinz means. Math. Inequal. Appl. 16, 93-99 (2013)

3. Zhan, X: Inequalities for unitarily invariant norms. SIAM J. Matrix Anal. Appl. 20, 466-470 (1998)

4. $\mathrm{Fu}, \mathrm{X}, \mathrm{He}, \mathrm{C}$ : On some inequalities for unitarily invariant norms. J. Math. Inequal. 7(4), $727-737$ (2013)

5. Kaur, R, Moslehian, MS, Singh, M, Conde, C: Further refinements of the Heinz inequality. Linear Algebra Appl. 447, 26-37 (2014)

6. He, CJ, Zou, LM, Qaisar, S: On improved arithmetric-geometric mean and Heinz inequalities for matrices. J. Math. Inequal. 6(3), 453-459 (2012)

7. Bakherad, M, Moslehian, MS: Reverses and variations of Heinz inequality. Linear Multilinear Algebra (2014). doi:10.1080/03081087.2014.880433

8. Bhatia, R, Sharma, R: Some inequalities for positive linear maps. Linear Algebra Appl. 436, 1562-1571 (2012)

9. Wang, S, Zou, L, Jiang, Y: Some inequalities for unitarily invariant norms of matrices. J. Inequal. Appl. 2011, 10 (2011)

doi:10.1186/1029-242X-2014-288

Cite this article as: Cao and Wu: Unitarily invariant norm inequalities involving Heron and Heinz means. Journal of Inequalities and Applications 2014 2014:288.

\section{Submit your manuscript to a SpringerOpen ${ }^{\circ}$ journal and benefit from:}

- Convenient online submission

Rigorous peer review

- Immediate publication on acceptance

- Open access: articles freely available online

- High visibility within the field

- Retaining the copyright to your article 